\title{
Hierarchies and Optima
}

\author{
Jean Serra \\ Université Paris-Est, 2, Bd Blaise Pascal, 931621 Noisy-le-Grand, France \\ j.serra@esiee.fr
}

\begin{abstract}
This paper is devoted to hierarchies of partitions, on which all criteria are proved to be connective. Optimisations are addressed by minimizing energies that satisfy the condition of hierarchical increasingness. The optimal cuts through the hierarchies are found. It is shown that many of the classical techniques are variants of what is proposed.
\end{abstract}

\section{Introduction: Optimum Optimorum}

Various segmentation algorithms base their approach on a stack of increasing partitions depending on a positive index. The stack, which has been obtained by a first wave of processing, serves as a framework for a second step, sometimes followed by a third one, and aims to lead to a final optimal partition of segmentation. There are several reasons to do so. Some regions of the image under study may require a finer treament than others. Then one starts from over segmentations which are recomposed [2], 18, [19]. Sometimes, one wants to reduce the size of an image while keeping its major features [16. Another reason appears with multivariate data, when one has to merge partitions coming from different bands 11. Sometimes also, the hierarchy is a direct consequence of the partitioning algorithm; a topological watershed, for example, produces a series of edges of a constant value, their saliencies [9] [11.

Most of these algorithms yield a unique final partition, though the meaning of the underlying optimization - if it exists- is rather unclear. A significant advance to clarify this point comes from L. Guigues et Al. [5] who introduced a linear decomposition of some energy over partitions. We will extend below the principle of their approach. After having decribed the structure of the hierarchies of partitions (section 2), we develop some theory about minimum cuts (section 3), which is illustrated by a few examples in section 4 .

\section{Hierarchies of Partitions}

From now on we suppose that a first step of segmentation already led to a finite hierarchy, i.e. to some finite sequence of increasing finite partitions. We will now intend to reorganize the classes of these partitions for extracting their quintessence, namely a more synthetic partition. Does the chain structure of the initial partitions provide us with particular assets for the second step of optimization?

I. Debled-Rennesson et al. (Eds.): DGCI 2011, LNCS 6607, pp. 35-46, 2011.

(C) Springer-Verlag Berlin Heidelberg 2011 


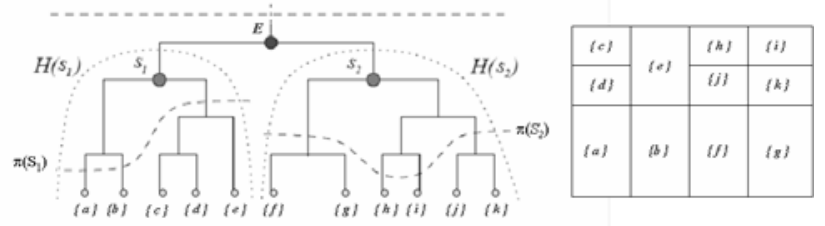

Fig. 1. Left, hierarchical tree; right, the corresponding space structure. $S_{1}$ and $S_{2}$ are the nodes sons of $E$, and $H\left(S_{1}\right)$ and $H\left(S_{1}\right)$ are the associated sub-hierarchies. $\pi_{1}$ and $\pi_{2}$ are cuts of $H\left(S_{1}\right)$ and $H\left(S_{1}\right)$ respectively, and $\pi_{1} \sqcup \pi_{2}$ is a cut of $E$.

\subsection{Reminder}

Finite hierarchies appeared initially in taxonomy for classifying objects. One can quote in particular the works of J.P. Benzécri [3] and of E. Diday [4]. We owe to the first author the theorem linking ultrametrics with hierarchies, which is involved below in Theorem 5. In image processing, hierarchical structures hold sometimes on stacks of images, but more often of operators, such as the classical families of Gaussian convolutions of D. Marr [7, or again the granulometries of G. Matheron [8. The hierarchies indicated in the introduction occupy an intermediary position, since they start from a given chain $H$ of segmentations of a fixed function $f$ on set $E$, i.e. from a stack of scalar or vector images, and this chain is then used as the framework for further operators. Here, for the sake of simplicity, we consider function $f$ and hierarchy $H$ as two starting points, possibly independent. This results in the following definition:

Definition 1. Let $\mathcal{D}$ be the set of all partitions of a finite set $E$, equipped with the refinement odrering $\ll$. A hierarchy $H$ of partitions $\pi_{i}$ is a finite chain in $\mathcal{D}$, i.e.

$$
H=\left\{\pi_{i}, 0 \leq i \leq n, \pi_{i} \in \mathcal{D} \mid i \leq j \Rightarrow \pi_{i} \ll \pi_{j}\right\},
$$

of extremities the extrema of $\mathcal{D}$, namely $\pi_{0}=\{\{x\}, x \in E\}$ and $\pi_{n}=\{E\}$.

Denote by $\mathcal{S}$ the set of all classes $S_{i}(x)$ of all partitions $\pi_{i}$ of $H$, i.e. $\mathcal{S}=$ $\left\{S_{i}(x), x \in E, 0 \leq i \leq n\right\}$. The expression (1) means that at each point $x \in E$ the family of those classes $S_{i}(x)$ of $\mathcal{S}$ that contain $x$ forms a finite chain $\mathcal{S}_{x}$ in $\mathcal{P}(E)$, of nested elements from $\{x\}$ to $E$ :

$$
\mathcal{S}_{x}=\left\{S_{i}(x), 0 \leq i \leq n\right\} .
$$

The finiteness $E$ is not really necessary. It mainly serves to ensure that the border lengths between classes are finite, but in 2D the Euclidean Voronoi model grants it as well. On the other hand, the search for minimal cuts by an induction on the hierarchical levels (Proposition 7), requires that their number is finite. Remark also that the parameter $i$ of the level can be replaced by any strictly increasing function $t(i)$, which defines another indexing of the hierarchy. 


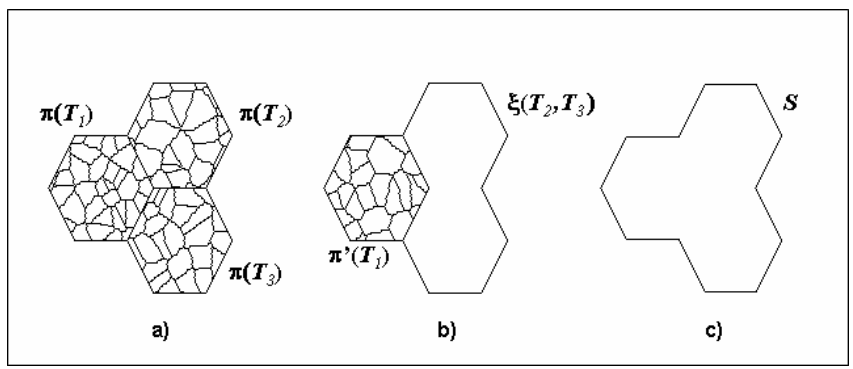

Fig. 2. The three hexagons a) are the three sons $T_{1}, T_{2}, T_{3}$ of summit $S$ (in c)). The concatenation $\pi\left(T_{1}\right) \sqcup \pi\left(T_{2}\right) \sqcup \pi\left(T_{3}\right)$ of three of their cuts is a cut of $S$ (a). Another cut of $S$ is obtained by taking $T_{1} \cup T_{2} \cup T_{3}=S$ (in c). However, $\pi^{\prime}\left(T_{1}\right) \sqcup \xi\left(T_{2}, T_{3}\right)$, in b), is not a valid cut of $S$.

According to a classical result, a family $\left\{S_{i}(x), x \in E, 0 \leq i \leq n\right\}$ of indexed sets generates the classes of a hierarchy iff

$$
\begin{gathered}
i \leq j \text { and } x, y \in E \Rightarrow S_{i}(x) \subseteq S_{j}(y) \\
\text { or } S_{i}(x) \supseteq S_{j}(y) \text { or } S_{i}(x) \cap S_{j}(y)=\emptyset .
\end{gathered}
$$

Conventionally, a hierarchy is represented by a tree where each node of bifurcation is a class $S$. The classes of $\pi_{i-1}$ at level $i-1$ which are included in $S_{i}(x)$ are said to be the sons of $S_{i}(x)$. Clearly, the sets of the descendants of each $S$ forms in turn a hierarchy $H(S)$ of summit $S$, which is included in the complete hierarchy $H=H(E)$. Figure 1 depicts two examples of such sub hierarchies, by the two zones $H\left(S_{1}\right)$ and $H\left(S_{2}\right)$ in small dotted lines.

\subsection{Cuts in a Hierarchy}

Any partition $\pi$ of $E$ whose classes are taken in $\mathcal{S}$ defines a cut in hierarchy $H$. The set of all cuts of $E$ is denoted by $\Pi(E)=\Pi$. Every "horizontal" section $\pi_{i}(H)$ at level $i$ is obviously a cut, but several levels can cooperate in a same cut, such as $\pi\left(S_{1}\right)$ and $\pi\left(S_{2}\right)$, drawn with thick dotted lines in Figure 1, Similarly, the partition $\pi\left(S_{1}\right) \sqcup \pi\left(S_{2}\right)$ generates a cut of $H(E)$. The symbol $\sqcup$ is used here for expressing that groups of classes are concatenated. Each class $S$ may also be the matter of cuts in the sub-hierarchy $H(S)$ whose $\mathcal{S}$ is the summit. Let $\Pi(S)$ be the family of all cuts of $H(S)$; put

$$
\widetilde{\Pi}=\cup\{\Pi(S), S \in \mathcal{S}\} .
$$

The set $\widetilde{\Pi}$ does not contain all possible partial partitions with classes in $\mathcal{S}$. For example, the partial partition $\{a\} \sqcup\{f\}$ of Figure 1 does not belong to $\widetilde{\Pi}$, though its two terms are members of $\mathcal{S}$. Moreover, all unions of classes are not in $\mathcal{S}$. If for example, $S$ is made by union of the three hexagonal sons $T_{1}, T_{2}, T_{3}$ 
(Figure 2), then the hierarchy $H(S)$ may accept a cut of type 2 a, but surely not of type $2 \mathrm{~b}$, because the union of two hexagons is not a class of $\mathcal{S}$. However, $\widetilde{\Pi}$ contains the family $\Pi(E)$ of all cuts of $H(E)$.

The hierarchical structure of the data induces a relation between the family $\Pi(S)$ of the cuts of node $S$ and the families $\Pi\left(T_{1}\right), \ldots, \Pi\left(T_{p}\right)$ of the sons $T_{1}, . ., T_{p}$ of $S$. Since all expressions of the form $\sqcup\left\{\pi\left(T_{k}\right) ; 1 \leq k \leq p\right\}$ define cuts of $S$, $\Pi(S)$ contains the whole family

$$
\Pi^{\prime}(S)=\left\{\pi\left(T_{1}\right) \sqcup . . \pi\left(T_{k}\right) . . \sqcup \pi\left(T_{p}\right) ; \quad \pi\left(T_{1}\right) \in \Pi\left(T_{1}\right) \ldots \pi\left(T_{p}\right) \in \Pi\left(T_{p}\right)\right\},
$$

plus the cut of $S$ into a unique class, i.e. $S$ itself, which is not a member of $\Pi^{\prime}(S)$. And as the other unions of several $T_{k}$ are not classes listed in $\mathcal{S}$, there is no other possible cut, hence

$$
\Pi(S)=\Pi^{\prime}(S) \cup S
$$

\subsection{Properties of the Hierarchies of Partitions}

The chain structure of the partitions of hierarchy $H$ gives rise to a few nice properties about lattices, connections and ultrametrics.

Lattices. The lattice structure results from the following property [17:

Proposition 2. The family $\Pi(E)$ of all cuts of $H$ is a complete lattice of partitions for the ordering of refinement, where the class of the infimum of $J$ cuts at point $x$ is the intersection of the classes in $x$ of the $J$ operands, and where the supremum is their union.

The fact that the union of the classes represents the class of the supremum derives from Proposition 13 in $[13$. The lattice $\Pi(E)$ is atomic. Its atoms are the partitions whose all classes but one are singletons, the last one being a pair of singletons.

Each increasing sequence $\{\pi(i), 0 \leq i \leq n\}$ of cuts whose classes come from $S$, and whose extremities are $\pi_{0}$ and $\pi_{n}$, defines in turn a new hierarchy $H^{\prime}$, different form $H$, but of the same lenght, and made from classes of $\mathcal{S}$, just as $H$ is. Let $\mathcal{H}$ stands for the family of all hierarchies of this type. The refinement ordering on the partitions induces a product ordering on the families of increasing cuts, i.e. on the hierarchies of $\mathcal{H}$, according to which $H_{1}$ is smaller than $H_{2}$ iff at every level $k$ the cut $\pi_{1}(k)$ is smaller than $\pi_{2}(k)$. We can state the following:

Corollary 3. Proposition 2 shows that the set $\mathcal{H}$ of all hierarchies having their classes in $\mathcal{S}$ is itself a lattice. All these lattices are finite, hence complete.

Connections. Concerning connective segmentation, the family $\mathcal{S}$ of all classes satisfies Proposition 4 and Theorem 5] [17:

Proposition 4. Every binary criterion $\sigma:(\mathcal{F}, \mathcal{S} \cup \varnothing) \rightarrow\{0,1\}$ is partially connective, or connective when it is satisfied by the singletons.

In other words, every subset of $\mathcal{S}$ containing $\emptyset$ is a partial connection. A similar result appears in the example 21 of [14. 
Ultrametrics. A distance is said to be ultrametric when the triangular inequality is replaced by the more severe following axiom [4, [3]:

$$
d(x, z) \leq \max \{d(x, y), d(y, z)\} .
$$

Two ultrametric balls can only be disjoint or concentric. Therefore equation (5) implies that the set of all balls of radius $r$ induces a Voronoi tesselation $\pi_{r}$ on $E$, whose classes are the open balls on the one hand, and the set of the frontiers on the other hand. The same equation (5) shows also that this Voronoi tesselation does not depend explicitly on a set of sites in space $E$, but only on radius $r$. When the range of variation of $r$ is finite, then the family $H=\left\{\pi_{r}, r \geq 0\right\}$ forms an indexed hierarchy. Conversely, every hierarchy of partitions determines an ultrametric over the set $\mathcal{S}$ of the classes of $H$ [3. Therefore, we can equivalently give ourselves an ultrametric or a hierarchy of partitions. This aspect is studied in detail by P. Arbelaez and L. Cohen in [2, by C. Ronse in [14, and by F. Meyer and L. Najman in 9 .

We can try and compare the disjunctive behavior of the ultrametric with the property of the classes of a hierarchy (Equation (2) ). Indeed, the three concepts of an indexed hierarchy, an ultrametric, and a universal connective criterion turn out to be three aspects of a same notion. More precisely, we have:

Theorem 5. The three following statements are equivalent:

1. $H$ is an indexed hierarchy,

2. the set $\mathcal{S}$ of all classes of the partition $\pi_{i}$ of $H$ forms an ultrametric space, of distance the indexing parameter,

3. every binary criterion $\sigma:(\mathcal{F}, \mathcal{S} \cup \varnothing) \rightarrow\{0,1\}$ is partially connective.

Proof. the implication $(1) \Rightarrow(3)$ is proved by proposition 4 , and the equivalence $(1) \Leftrightarrow(2)$ is a classical result [3]. We have to prove that $(3) \Rightarrow(1)$. We observe firstly that, given two sets $B_{1}$ and $B_{2}$, the criterion " $\sigma^{*}(f, A)=1$ iff $A \subseteq B_{1}$ or $A \subseteq B_{2}$ " is connective iff $B_{2} \subseteq B_{1}$ or $B_{1} \subseteq B_{2}$. Let then $H$ be a family of partitions. If it is not hierarchical then there exists at least one point $x$ included in two classes $B_{1}$ and $B_{2}$ of partitions of $H$ such that none of the two inclusions $B_{2} \subseteq B_{1}$ and $B_{1} \subseteq B_{2}$ is true, which implies that criterion $\sigma^{*}$ is not connective, which achieves the proof.

This result shows that the connective approach is inefficient for hierarchies, and orients us towards the alternative method, namely the optimization of an energy.

\section{Cuts of Minimum Energy in $\Pi(E)$}

We set the problem as it was formulated by L.Guigues [6] [5], and solved by him in the framework of additive energies and binary hierarchies. Below, the first of these two conditions is replaced by the more general relation (6) of hierarchical increasingness of the energy, and the second condition is shown to be cumbersome. In addition, the lack of unicity will lead us to analyze the structure of the solutions. 


\subsection{Minimization under Hierarchical Increasingness}

Allocate an energy $\omega$ over he set $\widetilde{\Pi}$ of partial partitions (Equation (3)), i.e. a positive numerical function $\omega: \widetilde{\Pi} \rightarrow \mathbb{R}$. We propose to characterize the cut(s) of $\Pi(E) \subseteq \widetilde{\Pi}$ of minimum energy. As family $\Pi(E)$ is finite, there is always at least one cut $\pi^{*}(E)$ of smallest energy. More generally, for each node $S$, the family $\Pi(S)$ of all cuts of the hierarchy of summit $S$ admits at least one minimum cut $\pi^{*}(S)$.

The search for minimum cuts becomes easier when we relate the energies of the fathers, $S$ say, to those of their sons, $T$ say, which can be obtained by means of hierarchical increasingness:

Definition 6. Let $H$ be a finite hierarchy, let $S$ be one of its nodes, and $T$ be one of the sons of $S$. An energy $\omega$ on the family $\widetilde{\Pi}$ of the cuts of $H$ satisfies the condition of hierarchical increasingness in $\widetilde{\Pi}$ when

$$
\omega\left(\pi_{1}(T)\right) \leq \omega\left(\pi_{2}(T)\right) \quad \Rightarrow \quad \omega\left[\left(\pi_{1}(T) \sqcup \pi(S \backslash T)\right] \leq \omega\left[\left(\pi_{2}(T) \sqcup \pi(S \backslash T)\right],\right.\right.
$$

where $\pi_{1}(T)$ and $\pi_{2}(T)$ are two cuts of the sub-hierarchy of summit $T$, and where $\pi$ is an arbitrary cut of the set difference $S \backslash T$.

The implication (6) extends an inequality relative to the partitions of set $T$ to another inequality holding on the partitions of set $S$, larger than $T$. In that, it is a matter for partial connective segmentation, in Ch. Ronse's sense [13].

Proposition 7. Let $H$ be a finite hierarchy, and $\omega$ be a hierarchically increasing energy on the cuts of $H$. If $S$ is a node of $H$ with $p$ sons $T_{1} . . T_{p}$ of minimum cuts $\pi_{1}^{*}, . . \pi_{p}^{*}$, then one of the two cuts

$$
\pi_{1}^{*} \sqcup \pi_{2}^{*} . . \sqcup \pi_{p}^{*},
$$

or the partition of $S$ into a unique class, is a minimum cut of $S$.

Proof. The hierarchical increasingness of the energy implies that cut (7) has the lowest energy among all the cuts of type $\Pi^{\prime}(S)=\sqcup\left\{\pi\left(T_{k}\right) ; 1 \leq k \leq p\right\}$ (it does not follow that it is unique). Now, from the decomposition (4), every cut of $S$ is either an element of $\Pi^{\prime}(S)$, or $S$ itself. Therefore, the set formed by the cut (77) and $S$ contains at least one minimum cut of $S$.

The condition of hierarchical increasingness (6) involved in Proposition 7 is not compulsory, but it opens a broad range of energies, and is easy to check. The analysis presented in [6] and [5] focuses on the case of the separable energies, i.e. of those that satisfy the additivity relation

$$
\omega\left(T_{1} \sqcup T_{2} . . \sqcup T_{p}\right)=\sum_{1 \leq k \leq p} \omega\left(T_{k}\right)
$$

between the energy of a partition and those of its classes. It is the concern of a particular hierarchical increasingness. But the algorithm proposed in 6] p.142, for finding minimum cuts in case of separable energies applies indeed to any hierarchically increasing energy $\omega$. It can be stated as follows: 
Proposition 8. Guigues'algorithm:

- scan in one pass all nodes of $H$ according to an ascending lexicographic order ;

- determine at each node $S$ a temporary minimum cut of $H$ by comparing the energy of $S$ to that of the concatenation of the temporary minimum cuts of the (already scanned) sons $T_{k}$ of $S$.

Remark that from Proposition [7, the temporary minimum holds always on two terms only, at each comparison, whatever the number of sons of $S$.

\subsection{Lattice of the Minimum Cuts}

The previous results never impose unicity for the minimum cuts. Each node $S$ involves its own series of comparisons, so that it can perfectly happen that in the family $\Pi(S)$ of Relation (4) a minimum cut of $\Pi^{\prime}(S)$ has the same energy as that of $S$. This event introduces two solutions which are then carried over the whole induction. And since such a doublet can occur regarding any node $S$ of hierarchy $H$, the number of minimal cuts is a priori indefinite, and may be large. However, one can reduce it by some additional condition, the simplest one being the ordering of refinement itself. Indeed, at each node $S$, among the two possible solutions of Proposition 7, the partition of $S$ into a single class is always the larger one for the refinement order. By ordering the solutions at each step, we thus structure them in a complete lattice whose cardinal increases according to thickness of quantification of the energy.

What advantage can we draw from this lattice structure? First of all, it solves the unicity problem. The question "find the cut that minimizes the energy" is replaced by "find the largest cut that minimizes the energy". This largest solution is characterized by the following proposition:

Proposition 9. Let $\{x\}$ be an element of the base of a hierarchy $H$ with $n+1$ levels, and let $S_{1}(x), S_{2}(x), . . S_{n}(x)$ be the sequence of the nodes anterior to $\{x\}$. Let $\omega$ be hierarchically increasing energy for the hierarchy on $\Pi(E)$, and $\pi^{*}(E)$ be a minimum cut $H(E)$ for this energy. The class of $\pi^{*}(E)$ that contains $\{x\}$ is then $S_{i}(x)$ such that

$$
\begin{gathered}
\omega\left[\pi^{*}\left(S_{i-1}(x)\right)\right] \geq \omega\left[\pi^{*}\left(S_{i}(x)\right)\right] \\
i \leq j<p \Rightarrow \omega\left[\pi^{*}\left(S_{j}(x)\right)\right]<\omega\left[\pi^{*}\left(S_{j+1}(x)\right)\right]
\end{gathered}
$$

Instead of using the refinement, we can, alternatively, introduce a second optimization. For example, for color images, $\omega$ can hold on the luminance, and the criterion for choosing between the optimal cuts can derive from the product saturation $\times$ hue.

\subsection{Minimization with External Partitions}

It may be sometimes advantageous to complete family $\widetilde{\Pi}$ of Relation (3) by additional partitions. For example, one can wish to suppress parasite small classes 
by clustering them with their neighbor. This leads to introduce a set $\Xi(S)$ of partial partitions of $S$ whose all classes are unions of more than one $T_{k}$, such as the class $\xi\left(T_{2}, T_{3}\right)$ made of the union of two hexagons in figure $2 \mathrm{~b}$. These supplementary cuts of $S$ generate the following family (up to a permutation of the indexes)

$$
\Pi^{\prime \prime}(S)=\left\{\pi\left(T_{1}\right) \sqcup . . \pi\left(T_{k}\right) \sqcup \xi\left(T_{k+1} . . T_{p}\right) \quad 1 \leq k \leq p ; \xi\left(T_{k+1} . . T_{p}\right) \in \Xi(S)\right\} .
$$

Family (11) has to be added to that, $\Pi(S)$, of the legitimate sons (Relation (4)). Proposition 7 admits now the new formulation [17]

Proposition 10. Let $S$ be a node in hierarchy $H$, of $p$ sons $T_{1} . . T_{p}$ associated with $p$ minimum cuts $\pi_{1}^{*}, . . \pi_{p}^{*}$, for some increasing energy. The minimum cuts of the set formed by

i) the cut

$$
\pi_{1}^{*} \sqcup \pi_{2}^{*} . . \sqcup \pi_{p}^{*},
$$

ii) the family

$$
\left\{\pi_{1}^{*} \sqcup \pi_{2}^{*} . . \sqcup \pi_{k}^{*} \sqcup \xi\left(T_{k+1}, . . T_{p}\right) ; \xi \in \Xi(S)\right\},
$$

contains the minimum cuts of $S$.

Proposition 10 reduces the number of comparisons to do for finding the minimum cut of $H(S)$ from those of the sons of $S$. To illustrate that, we can calculate how many comparisons are necessary when $S$ has 2,3 or 4 sons. For two sons $T_{1}$ and $T_{2}$, it suffices to compare the energies of the two terms $\pi_{1}^{*} \sqcup \pi_{2}^{*}$ and $S=T_{1} \cup T_{2}$. When a third son $T_{3}$ is added, we go to five terms $\pi_{1}^{*} \sqcup \pi_{2}^{*} \sqcup \pi_{3}^{*}, T_{1} \cup T_{2} \cup T_{3}$, plus the three cuts of type $\pi_{1}^{*} \sqcup \xi\left(T_{2}, T_{3}\right)$ (see Figure 2). For the nodes with four sons one finds, similarly, $1+C_{4}^{2}+C_{4}^{3}+4=15$ possibilities. Note that all these values can only reduce when we require also that the classes of partitions $\xi$ must be connected. Finally, the previous algorithm in one pass remains valid, but more comparisons must be performed at each node. But now the solutions no longer form a lattice, which is an obvious drawback.

\section{Examples of Hierarchical Minimizations}

We go back over the studies quoted in introduction, and try and interpret them in the framework that has just been developed. These studies are obviously richer than their specifics aspects of hierarchical optimization on which we concentrate here.

\subsection{Suprema of Energies}

We firstly contemplate energies whose law of composition is similar to the additivity relation (8), but written for the supremum. If $T_{1}, . . T_{k}, . . T_{p}$, stand for the classes of partition $\pi$, we thus have

$$
\omega\left(T_{1} \sqcup T_{2}, . . \sqcup T_{p}\right)=\sup \left\{\omega\left(T_{k}\right), 1 \leq k \leq p\right\} .
$$


The energies involved in the law (14) increase hierarchically, hence lend themselves to the above optimizations. Here are two examples, where we suppose also that the energy is an increasing function of the class, i.e.

$$
T \subseteq S \Rightarrow \omega(T) \leq \omega(S) \quad T, S \in \mathcal{S} .
$$

This last condition may seem to contradict the hierarchical rise, since the partition into singletons, from which we start, becomes a solution. But it makes sense when we quantify the energy in 0 and 1 , if we look for the largest element in the lattice of all solutions.

Lasso. This algorithm, due to F. Zanoguera et Al. [20], appears also in [9]. The initial image is first segmented into $\alpha$-flat zones, which generate a hierarchy as the slope $\alpha$ increases. But the optimization itself applies to any hierarchy of segmentations. It consists in drawing manually a closed curve around the object to segment. If $A$ designates the inside of this lasso, then we take the following function

$$
\begin{array}{ll}
\omega(S)=0 & \text { when } S \subseteq A ; S \in \mathcal{S} \\
\omega(S)=1 & \text { when not, }
\end{array}
$$

for energy, and we go from classes to partitions by the law (14) of $\vee$-composition of the energies. The largest cut that minimizes $\omega$ is depicted in Figure 3 . We see that the resulting contour follows the edges of the petals. Indeed, a segmented class can jump over this high gradient only for $\alpha$ large enough, and then this class is rejected because it spreads out beyond the limit of the lasso.

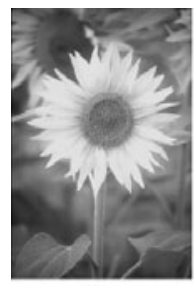

a)

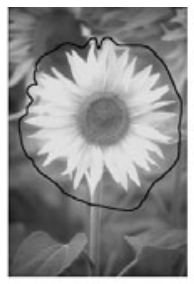

b)

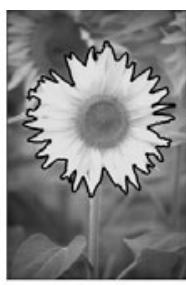

c)

Fig. 3. a) Initial image; b) manual lasso; c) contour of the union of the classes inside the lasso

Segmentation under constraint. This method was proposed by P. Soille and J. Grazzini in 18 and 19 with several variants; it is re-formulated by C. Ronse in a more general framework in [15]. We now take for energy $\omega$ of the classes the function

$$
\begin{array}{ll}
\omega(S)=0 & \text { when } \sup \{f(x), x \in S\}-\inf \{f(x), x \in S\} \leq \omega_{0} \\
\omega(S)=1 & \text { when not, }
\end{array}
$$

where $\omega_{0}$ is a given threshold, and we go to partitions by $\vee$-composition. In the examples of [18] and [19], the hierarchy is obtained by $\alpha$-flat zones, and, 
for multi-spectral images, by the intersection of the $\alpha$-flat zones of the various bands. Sometimes the criterion "there is at least one extremum of $f$ inside $S$ " is added. The class at point $x$ of the largest partition of minimum energy is given by the largest $S \in \mathcal{S}$, that contains $x$, and such that the amplitude of variation of $f$ inside $S$ be $\leq \omega_{0}$.

\subsection{Additive Energies}

Separable models. They are energies whose archetype is given by the classical Mumford and Shah functional. One constructs a separable energy in $\mathbb{R}^{2}$ or in $\mathbb{Z}^{2}$ by starting from a partition of $S$ into its sons $T_{1}, . . T_{p}$ plus the set $\Gamma$ of their frontiers. A first measure $\omega_{\mu}$ holds on the $T_{k}$, and a second one, $\omega_{\nu}$, on their frontiers $\partial T_{k}$, and we put

$$
\omega(S)=\sum_{1 \leq k \leq p} \omega_{\mu}\left(T_{k}\right)+\omega_{\nu}\left(\partial T_{k}\right),
$$

(according to Integral Geometry, a third term, proportional to Euler-Poincaré Constant could be added). This models are said to be separable [6] [5] in that we have $\omega(S)=\omega\left(T_{1}\right)+\ldots+\omega\left(T_{p}\right)$, up to external edge effects. The energy $\omega$, additive, is thus hierarchically increasing. Different instructive variants may be found in [6].

Thumbnails. The creation of digital thumbnails by Ph. Salembier and L. Garrido [16] is a matter for separable models. One aims to generate reduced versions of a given color image $f$ from a hierarchy of its segmentations. In each class $T$ function $f$ is replaced by its mean value $m(T)$. The approximation is estimated in the $L_{2}$ norm, i.e.

$$
\omega_{\mu}(T)=\sum_{x \in R}\|f(x)-m(T)\|^{2} .
$$

If the coding cost for a frontier element is $k$, that of the whole class $T$ becomes

$$
\omega_{\nu}(T)=24+\frac{k}{2}|\partial T|
$$

with 24 bits for $m(T)$. The total energy of a cut is written $\omega(S)=\lambda \omega_{\mu}(S)+\omega_{\nu}(S)$. For a fixed $\lambda$, it yields a first minimization, followed by a second one relatively to the Lagrange parameter $\lambda$.

\subsection{Other Laws of Composition}

A number of laws of composition are compatible with hierarchically increasing energies. Instead of supremum and sum, that we just presented, one could use infimum, product, difference sup-inf, quadratic sum, etc., and all their combinations. Moreover, one can make depend $\omega$ on more than one class, on the proximity of the edges, on several elements of the lattice $\mathcal{H}$ of the hierarchies, etc... 
Here is an example of another law of composition, which extends the technique developed in [1]. Start from three partition hierarchies $H_{l}, H_{s}$, and $H_{h}$ of luminance, saturation, and hue of a given color image. The purpose is to reduce them to a unique significant cut, and the idea is that the higher (resp. lower) the saturation, the more representative is the hue (resp. luminance). Therefore, when a region has a weak (resp. strong) saturation, then its luminance (hue) is prioritary for the segmentation. Suppose that saturation $s$ varies between 0 and 1. Provide the classes of $H_{s}$ with the energy

$$
\omega\left(T_{s}\right)=\frac{1}{\text { area } T_{s}}\left[1-\int_{T_{s}} s(x) d x\right],
$$

and weight by areas for expressing the energies of the partitions from those of the classes. It results in the minimum cut $\bar{\pi}_{s}$. Repeat the process by replacing $1-\int_{T_{s}} s(x) d x$ by $\int_{T_{s}} s(x) d x$ in (17), which results in the new energy $\omega^{\prime}\left(T_{s}\right)$ and the new minimum cut $\bar{\pi}_{s}^{\prime}$. Intersect then the two partitions $\bar{\pi}_{s}$ and $\bar{\pi}_{s}^{\prime}$. When the class at point $x$ of the intersection has an energy $\omega-\omega^{\prime}>0$, it is labelled "class for luminance", and when not, "class for hue". The process ends by taking the partial hierarchy of the luminance (resp.the hue) in the union of the classes for luminance (resp. for hue), and segmenting luminance and hue individually, in their own domains.

\section{Conclusion}

Considering the hierarchies of partitions of an image as a segmentation tool, we looked for the meaning of an optimum cut, for laws able to govern this optimum. We showed that the assumption of hierarchical increasingness (6) allows to regroup various segmentation techniques which seem to be far away from each other, and we proposed new ones.

Acknowledgement. The author wishes to thank J. Angulo, J. Cousty, L. Najman, and Ch. Ronse for their valuable comments.

\section{References}

1. Angulo, J., Serra, J.: Modeling and segmentation of colour images in polar representations. Image and Vision Computing 25, 475-495 (2007)

2. Arbelaez, P., Cohen, L.D.: Segmentation d'images couleur par partitions de Voronoï. Traitement du Signal 21(5), 407-421 (2004)

3. Benzécri, J.P.: L'Analyse des Données. Tome I: La Taxinomie, Dunod, Paris, 4e édition (1984)

4. Diday, E., Lemaire, J., Pouget, J., Testu, F.: Eléments d'analyse des données, Dunod, Paris (1982)

5. Guigues, L., Cocquerez, J.P., Le Men, H.: Scale-Sets Image Analysis. Int. Journal of Computer Vision 68(3), 289-317 (2006)

6. Guigues, L.: Modèles multi-échelles pour la segmentation d'images. Thèse doctorale Université de Cergy-Pontoise, décembre (2003) 
7. Marr, D.: Vision. Freeman and Co., New York (1982)

8. Matheron, G.: Random Sets and Integral Geometry. Wiley, New-York (1975)

9. Meyer, F., Najman, L.: Segmentation, Minimum Spanning Trees, and Hierarchies. In: Najman, L., Talbot (eds.) Mathematical Morphology. Wiley, N.Y. (2010)

10. Najman, L.: Ultrametric watersheds. In: Wilkinson, M.H.F., Roerdink, B.T.M. (eds.) ISMM 2009. LNCS, vol. 5720, pp. 181-192. Springer, Heidelberg (2009)

11. Najman, L.: Ultrametric watersheds: a bijection theorem for hierarchical edgesegmentation. In: JMIV (to appear, 2011)

12. Noyel, G., Angulo, J., Jeulin, D.: On distances, paths and connections for hyperspectral image segmentation. In: Proceedings of the 8th International Symposium on Mathematical Morphology, Rio de Janeiro, Brazil, October 10-13. MCT/INPE, vol. 1, pp. 399-410 (2007)

13. Ronse, C.: Partial partitions, partial connections and connective segmentation. Journal of Mathematical Imaging and Vision 32, 97-125 (2008)

14. Ronse, C.: Adjunctions on the lattices of partitions and of partial partitions. AAECC 21(5), 343-396 (2010)

15. Ronse, C.: Idempotent block splitting on partial partitions, I: isotoneoperators, II: non-isotone operators Order (to appear, 2011)

16. Salembier, P., Garrido, L.: Binary Partition Tree as an Efficient Representation for Image Processing, Segmentation, and Information Retrieval. IEEE Trans. on Image Processing 9(4), 561-576 (2000)

17. Serra, J.: Ordre de la construction et segmentations hié rarchiques. Colloque ESIEE (April 2, 2010), http://laurentnajman.org/serra70/index

18. Soille, P.: Constrained connectivity for hierarchical image partitioning and simplification. IEEE Transactions on Pattern Analysis and Machine Intelligence 30, 1132-1145 (2008)

19. Soille, P., Grazzini, J.: Constrained Connectivity and Transition Regions. In: Wilkinson, M.H.F., Roerdink, B.T.M. (eds.) Mathematical Morphlology and its Applications to Signal and Image Processing, pp. 59-70. Springer, Heidelberg (2009)

20. Zanoguera, F., Marcotegui, B., Meyer, F.: A toolbox for interactive segmentation based on nested partitions. In: Proc. of ICIP 1999, Kobe, Japan (1999) 